EESTI NSV TEADUSTE AKADEEMIA TOIMETISED. XIV KÖIDE

FOUSIKA-MATEMAATIKA- JA TEHNIKATEADUSTE SEERIA. 1965, NR. 2

ИЗВЕСТИЯ АКАДЕМИИ НАУК ЭСТОНСКОЙ ССР. ТОМ ХІV

ЄЕРИЯ ФИЗИКО:МАТЕМАТИЧЕСКИХ И ТЕХНИЧЕСКИХ НАУК. 1965, № 2

Ю. ЛЕМБРА

\title{
О ВЫЧИСЛЕНИИ КВАДРАТА МОДУЛЯ ФУНКЦИИ ФЛОКЕ УРАВНЕНИЯ БЕТАТРОННЫХ КОЛЕБАНИЙ В СЕКТОРНОМ ЦИКЛОТРОНЕ
}

В работе [1] мы применяли метод огибающих $[2,3]$ для исследования фокусирующих свойств секторного циклотрона $[4-6]$, элемент периодичности магнитной системы которого состоит из двух секторов с различными значениями напряженности однородного магнитного поля: $H$ (сектор 1) и $\chi H$ (сектор 2). Поскольку поведение огибающей по существу определяется поведением квадрата модуля функции Флоке (в дальнейшем сокращенно к.м.ф.Ф.) уравнения бетатронных колебаний, то в [1] наше внимание было обращено на исследование этой функции. Целью настояцей работы является представление к.м.Ф.Ф. с помощью нового метода, описанного в работе [7], в более простой форме, чем в [1], а также обобщение полученных результатов на случай слабо неоднородного магнитного поля.

Выпишем основные формулы для применения метода [7]. Бетатронные колебания описываются уравнением типа

$$
\frac{d^{2} u}{d s^{2}}+g(s) u=0 ; \quad g(s+L)=g(s) .
$$

Здесь $u=\varrho$ или $z$; $\varrho$ и $z$ - радиальное и вертикальное отклонения частицы от равновесной орбиты; $s$ - длина дуги равновесной орбиты; $L-$ длина элемента периодичности, измеренная вдоль равновесной орбиты.

Согласно [7], к.м.ф.Ф. Ф(s) уравнения (1) выражается формулой

$$
\Phi(s)=\frac{\mathfrak{A}_{12}(s)}{\sin \mu},
$$

где $\mathfrak{A}(s)$ - матрица элемента периодичности с началом в точке $s:$

$$
\mathfrak{U}(s)=\alpha(s) A \alpha^{-1}(s) .
$$

В формуле (3)

$$
\left.\begin{array}{rl}
\alpha(s) & =\left(\begin{array}{ll}
\alpha_{11}(s) & \alpha_{12}(s) \\
\alpha_{21}(s) & \alpha_{22}(s)
\end{array}\right) \\
\alpha_{21} & =\frac{d \alpha_{11}}{d s}, \quad \alpha_{22}=\frac{d \alpha_{12}}{d s} \\
A & =\alpha(L) .
\end{array}\right\}
$$


Здесь $\alpha_{11}$ и $\alpha_{12}$ - два линейно независимых решения уравнения (1) в интервале $0 \leqslant s \leqslant L$, удовлетворяющие начальным условиям

$$
\alpha(0)=\left(\begin{array}{ll}
1 & 0 \\
0 & 1
\end{array}\right) .
$$

Величина $\mu$, входящая в (2), определяется так:

$$
\left.\begin{array}{l}
\cos \mu=\frac{1}{2} \operatorname{Sp} A=\frac{1}{2} \operatorname{Sp} \mathfrak{U}(s) \\
(\sin \mu)=\operatorname{sgn} A_{12}=\operatorname{sgn} \mathfrak{U}_{12}(s) .
\end{array}\right\}
$$

\section{1. Радиальные колебания}

Согласно ['], можно для уравнения (1) положить:

$$
g_{r}(s)=\left\{\begin{array}{c}
\frac{1}{r^{2}} \text { в секторе } 1 \\
-\frac{1}{r_{21}^{2}} \text { в пз пектодной 1 в сектор } 2 \\
\frac{x^{2}}{r^{2}} \text { в секторе } 2 \\
-\frac{1}{r_{12}^{2}} \text { в пз пектора 2 в сектор 1. }
\end{array}\right\}
$$

В уравнении (7) $r$ - радиус кривизны равновесной орбиты в секторе 1. $r_{i j}=\sqrt{r l_{i j} /(1-x) \operatorname{tg} \gamma_{i j}}, l_{i j} \ll L(i \neq j ; i, j=1 ; 2), l_{12}$ и $l_{21}$ - длины переходных областей, измеренные вдоль равновесной орбиты, $\gamma_{12}$ и $\gamma_{21}$ - углы между касательной к равновесной орбите и нормалью к граничной линии, отделяющей сектор от переходной области. Знаки углов $\gamma_{12}$ и $\gamma_{21}$ определяем по принятому в [ $\left.{ }^{4}\right]$ правилу.

Поскольку по (7) функция $g_{r}(s)$ в данном случае кусочно-постоянная, удобно описывать бетатронные колебания по участкам элемента периодичности (сектор или переходная область) и ввести отсчет длины на каждом участке от своего начала.

Удобно ввести матрицы

$$
\left.\begin{array}{l}
U(a, x)=\left(\begin{array}{cc}
\cos a x & \frac{1}{a} \sin a x \\
-a \sin a x & \cos a x
\end{array}\right) \\
V(a, x)=\left(\begin{array}{cc}
\operatorname{ch} a x & \frac{1}{a} \operatorname{sh} a x \\
a \operatorname{sh} a x & \operatorname{ch} a x
\end{array}\right),
\end{array}\right\}
$$


причем

$$
\left.\begin{array}{l}
U(a, x) U(a, y)=U(a, x+y) \\
V(a, x) V(a, y)=V(a, x+y) .
\end{array}\right\}
$$

Очевидно в секторе 1 двумя линейно независимыми решениями уравнения (1) являются $\cos \frac{s}{r}$ и $\sin \frac{s}{r}$, из которых матрица $\alpha_{r}(s)$, обладающая свойством (5), может быть построена так:

$$
\alpha_{r}(s)=U\left(\frac{1}{r}, s\right) \quad\left(0 \leqslant s \leqslant r v_{1}\right)
$$

$\left(v_{1}-\right.$ угол поворота равновесной орбиты в секторе 1$)$.

Аналогично получим:

в переходной области из сектора 1 в сектор 2

$$
\alpha_{r}(s)=V\left(\frac{1}{r_{21}}, s\right) \quad\left(0 \leqslant s \leqslant l_{21}\right),
$$

в секторе 2

$$
\alpha_{r}(s)=U\left(\frac{x}{r}, s\right) \quad\left(0 \leqslant s \leqslant \frac{r v_{2}}{x}\right)
$$

( $v_{2}$ - угол поворота равновесной орбиты в секторе 2),

в переходной области из сектора 2 в сектор 1

$$
\alpha_{r}(s)=V\left(\frac{1}{r_{12}}, s\right) \quad\left(0 \leqslant s \leqslant l_{12}\right) .
$$

Перейдем к нахождению матриц $\mathfrak{A}_{r}(s)$. При получении $\mathfrak{A}_{r}(s)$ в секторе $1\left(0 \leqslant s \leqslant r v_{1}\right)$ следует под $A_{r}$ в выражении (3) рассматривать матрицу элемента периодичности, начало которой совпадает с началом сектора 1. Учитывая, что известны решения (10) - (13), можем матрицу $A_{r}$ легко получить методом «сшивания»:

$$
A_{r}=V\left(\frac{1}{r_{12}}, l_{12}\right) U\left(\frac{x}{r}, \frac{r v_{2}}{x}\right) V\left(\frac{1}{r_{21}}, l_{21}\right) U\left(\frac{1}{r}, r v_{1}\right) .
$$

Подставляя (10) и (14) в (3), найдем с учетом (9) для матрицы $\mathfrak{A},(s)$ в секторе 1 выражение

$$
\begin{array}{r}
\mathfrak{A}_{r}(s)=U\left(\frac{1}{r}, s\right) V\left(\frac{1}{r_{12}}, l_{12}\right) U\left(\frac{x}{r}, \frac{r v_{2}}{x}\right) V\left(\frac{1}{r_{21}}, l_{21}\right) U\left(\frac{1}{r}, r v_{1}-s\right) \\
\left(0 \leqslant s \leqslant r v_{1}\right) .
\end{array}
$$

Аналогично получим:

в переходной области из сектора 1 в сектор 2

$$
\begin{array}{ll}
\mathfrak{U}_{r}(s)=V\left(\frac{1}{r_{21}}, s\right) U\left(\frac{1}{r}, r v_{1}\right) V\left(\frac{1}{r_{12}}, l_{12}\right) U\left(\frac{\varkappa}{r}, \frac{r v_{2}}{\varkappa}\right) V\left(\frac{1}{r_{21}}, l_{21}-s\right) & \left(0 \leqslant s \leqslant l_{21}\right), \\
\text { В секторе } 2 &
\end{array}
$$




$$
\begin{array}{r}
\mathfrak{A}_{r}(s)=U\left(\frac{x}{r}, s\right) V\left(\frac{1}{r_{21}}, l_{21}\right) U\left(\frac{1}{r}, r v_{1}\right) V\left(\frac{1}{r_{12}}, l_{12}\right) U\left(\frac{x}{r}, \frac{r v_{2}}{x}-s\right) \\
\left(0 \leqslant s \leqslant \frac{r v_{2}}{x}\right),
\end{array}
$$

в переходной области из сектора 2 в сектор 1

$$
\begin{array}{r}
\mathfrak{A}_{r}(s)=V\left(\frac{1}{r_{21}}, s\right) U\left(\frac{x}{r}, \frac{r v_{2}}{x}\right) V\left(\frac{1}{r_{21}}, l_{21}\right) U\left(\frac{1}{r}, r v_{1}\right) V\left(\frac{1}{r_{12}}, l_{12}-s\right) \\
\left(0 \leqslant s \leqslant l_{12}\right) .
\end{array}
$$

Далее переходим, как и в [1], к рассмотрению случая, когда длины переходных областей стремятся к нулю, т. е. $l_{12} \rightarrow 0$ и $l_{21} \rightarrow 0$. Тогда матрицы $\mathfrak{A}_{r}(s)$ принимают форму:

в секторе 1

$$
\begin{array}{r}
\mathfrak{A}_{r}(s)=U\left(\frac{1}{r}, s\right) B\left(\frac{\tau_{12}}{r}\right) U\left(\frac{x}{r}, \frac{r v_{2}}{\chi}\right) B\left(\frac{\tau_{21}}{r}\right) U\left(\frac{1}{r}, r v_{1}-s\right) \\
\left(0 \leqslant s \leqslant r v_{1}\right),
\end{array}
$$

в секторе 2

$$
\begin{array}{r}
\mathfrak{A}_{r}(s)=U\left(\frac{x}{r}, s\right) B\left(\frac{\tau_{21}}{r}\right) U\left(\frac{1}{r}, r v_{1}\right) B\left(\frac{\tau_{12}}{r}\right) U\left(\frac{x}{r}, \frac{r v_{2}}{\chi}-s\right) \\
\left(0 \leqslant s \leqslant \frac{r v_{2}}{\varkappa}\right),
\end{array}
$$

где $B(x)=\left(\begin{array}{ll}1 & 0 \\ x & 1\end{array}\right)$ и $\tau_{i j}=(1-x) \operatorname{tg} \gamma_{i j}$.

Теперь удобно ввести матрицу

$$
\mathfrak{U}^{\prime}(s)=U\left(\frac{m_{1} k_{1}}{r}, s\right) B\left(\frac{t_{12}}{r}\right) U\left(\frac{m_{2} k_{2}}{r}, \frac{\Theta_{2} r}{k_{2}}\right) B\left(\frac{t_{21}}{r}\right) U\left(\frac{m_{1} k_{1}}{r}, \frac{r \Theta_{1}}{k_{1}}-s\right) .
$$

Матрицы в (19) и (20) можно получить из (21) после очевидных подстановок, показанных в табл. 1.*

Таблица 1

\begin{tabular}{ll|c|c|c|c|c|c|c|c}
\hline$m_{1}$ & $k_{1}$ & $m_{2}$ & $k_{2}$ & $t_{12}$ & $t_{21}$ & $\Theta_{1}$ & $\Theta_{2}$ \\
\hline Ceктор 1 & 1 & 1 & 1 & $x$ & $\tau_{12}$ & $\tau_{21}$ & $v_{1}$ & $v_{2}$ \\
\hline Ceктор 2 & 1 & $x$ & 1 & 1 & $\tau_{21}$ & $\tau_{12}$ & $v_{2}$ & $v_{1}$
\end{tabular}

* Целесообразность введения величин $m_{1}$ и $m_{2}$ выясняется в дальнейшем. 
Выполняя умножение матриц в (21) и производя несложные тригонометрические преобразования, получим:

$\mathfrak{A}_{12}^{\prime}=\frac{r}{2 m_{1} k_{\mathbf{x}}}\left\{\left[\left(t_{12}+t_{21}\right) \cos m_{2} \Theta_{2}+\left(m_{1}^{2} k_{1}^{2}-m_{2}^{2} k_{2}^{2}+t_{12} t_{21}\right) \frac{\sin m_{2} \Theta_{2}}{m_{2} k_{2}}\right] \times\right.$

$\times \frac{\cos m_{1}\left(\frac{2 k_{1} s}{r}-\Theta_{1}\right)}{m_{1} k_{1}}+\left(t_{12}-t_{21}\right) \frac{\sin m_{2} \Theta_{2}}{m_{2} k_{2}} \sin m_{1}\left(\frac{2 k_{1} s}{r}-\Theta_{1}\right)+$

$+\left[-\left(t_{12}+t_{21}\right) \cos m_{2} \Theta_{2}+\left(m_{1}^{2} k_{1}^{2}+m_{2}^{2} k_{2}^{2}-t_{12} t_{21}\right) \frac{\sin m_{2} \Theta_{2}}{m_{2} k_{2}}\right] \frac{\cos m_{1} \Theta_{1}}{m_{1} k_{1}}+$

$$
\left.+\left[2 \cos m_{2} \Theta_{2}+\left(t_{12}+t_{21}\right) \frac{\sin m_{2} \Theta_{2}}{m_{2} k_{2}}\right] \sin m_{1} \Theta_{1}\right\}
$$

$\frac{1}{2} \mathrm{Sp} \mathfrak{A}^{\prime}=\cos m_{1} \Theta_{1} \cos m_{2} \Theta_{2}-\frac{1}{2}\left(\frac{m_{1} k_{1}}{m_{2} k_{2}}+\frac{m_{2} k_{2}}{m_{1} k_{1}}\right) \sin m_{1} \Theta_{1} \sin m_{2} \Theta_{2}+$

$$
+\frac{t_{12}+t_{21}}{2}\left(\frac{\cos m_{1} \Theta_{1} \sin m_{2} \Theta_{2}}{m_{2} k_{2}}+\frac{\sin m_{1} \Theta_{1} \cos m_{2} \Theta_{2}}{m_{1} k_{1}}\right)+
$$

$\dashv \frac{t_{12} t_{21}}{2 m_{1} m_{2} k_{1} k_{2}} \sin m_{1} \Theta_{1} \sin m_{2} \Theta_{2}$.

Пользуясь табл. 1, найдем по (2) и (22) для к.м.ф.Ф. уравнения радиальных бетатронных колебаний следующее выражение:

в секторе 1

$$
\Phi_{r 1}(s)=\frac{r}{2 \sin \mu_{r}} \times
$$

$X\left\{\left[\left(\tau_{12}+\tau_{21}\right) c_{2}+\left(1-x^{2}+\tau_{12} \tau_{21}\right) \frac{s_{2}}{x}\right] \cos \left(\frac{2 s}{r}-v_{1}\right)+\right.$

$+\left(\tau_{12}-\tau_{21}\right) \frac{s_{2}}{\varkappa} \cdot \sin \left(\frac{2 s}{r}-v_{1}\right)+\left[-\left(\tau_{12}+\tau_{21}\right) c_{2}+\right.$

$\left.+\left(1+x^{2}-\tau_{12} \tau_{21}\right) \frac{s_{2}}{x}\right] c_{1}+\left[2 c_{2}+\left(\tau_{12}+\tau_{21}\right) \frac{s_{2}}{x}\right] s_{1}$;

в секторе 2

$$
\left(0 \leqslant s \leqslant r v_{1}\right) \text {, }
$$

$=\frac{r}{2 \varkappa \sin \mu_{r}}\left\{\frac{1}{x}\left[\left(\tau_{12}+\tau_{21}\right) c_{1}+\left(x^{2}-1+\tau_{12} \tau_{21}\right) s_{1}\right] \cos \left(\frac{2 \varkappa s}{r}-v_{2}\right)+\right.$

$+\left(\tau_{21}-\tau_{12}\right) s_{1} \sin \left(\frac{2 x s}{r}-v_{2}\right)+\left[-\left(\tau_{12}+\tau_{21}\right) c_{1}+\right.$

$\left.\left.+\left(x^{2}+1-\tau_{12} \tau_{21}\right) s_{1}\right] \frac{c_{2}}{\varkappa}+\left[2 c_{1}+\left(\tau_{12}+\tau_{21}\right) s_{1}\right] s_{2}\right)$

$$
\left(0 \leqslant s \leqslant \frac{r v_{2}}{x}\right)
$$

где $\quad c_{i}=\cos v_{i}, \quad s_{i}=\sin v_{i} \quad(i=1 ; 2)$.

5 ENSV TA Toimetised F-2 65. 
Для определения величины $\mu_{r}$, входящей в (23), согласно (6) и (22), остается справедливым известное из $[1,4-6]$ выражение

$$
\cos \mu_{r}=c_{1} c_{2}-\frac{1+\varkappa^{2}}{2 \varkappa} s_{1} s_{2}+\frac{\tau_{12}+\tau_{21}}{2}\left(s_{1} c_{2}+\frac{c_{1} s_{2}}{\varkappa}\right)+\frac{\tau_{12} \tau_{21} s_{1} s_{2}}{2 \varkappa} .
$$

\section{2. Вертикальные колебания}

Согласно [], можно для уравнения (1) положить:

$$
g_{z}(s)=\left\{\begin{array}{ll}
0 & \text { в секторе 1 } \\
\frac{1}{r_{21}^{2}} & \begin{array}{l}
\text { в переходной облора 1 в сектор 2 } \\
0
\end{array} \\
\frac{1}{r_{12}^{2}} & \begin{array}{l}
\text { в секторе } 2 \\
\text { нз сектора 2 в сектор 1. }
\end{array}
\end{array}\right\}
$$

В связи с тем, что расчеты вполне аналогичны проведенным в разделе 1 , мы на них подробно здесь не останавливаемся.

Для матрицы $\mathfrak{H}_{z}(s)$ имеем выражения:

в секторе 1

$$
\begin{aligned}
& \mathfrak{A}_{z}(s)=U(0, s) B\left(-\frac{\tau_{12}}{r}\right) U\left(0, \frac{r v_{2}}{x}\right) B\left(-\frac{\tau_{21}}{r}\right) U\left(0, v_{1} r-s\right) \\
& \text { в секторе } 2
\end{aligned}
$$

$\mathfrak{A}_{z}(s)=U(0, s) B\left(-\frac{\tau_{21}}{r}\right) U\left(0, r v_{1}\right) B\left(-\frac{\tau_{12}}{r}\right) U\left(0, \frac{v_{2} r}{\varkappa}-s\right)$

$$
\left(0 \leqslant s \leqslant \frac{r v_{2}}{x}\right) \text {. }
$$

Сравнение формул (21) и (26) показывает, что в данном случае в выражении $\mathfrak{U}^{\prime}(s)$ следует сделать подстановку (табл. 2).

Таблица 2

\begin{tabular}{l|c|c|c|c|c|c|c|c}
\hline Ceктор 1 & 0 & 1 & 0 & $\kappa$ & $-\tau_{12}$ & $-\tau_{21}$ & $v_{1}$ & $v_{2}$ \\
\cline { 2 - 7 } Ceктор 2 & 0 & $\varkappa$ & 0 & 1 & $-\tau_{21}$ & $-\tau_{12}$ & $v_{2}$ & $v_{1}$
\end{tabular}

Тогда получим из (2) и (22) для к.м.ф.Ф. уравнения вертикальных бетатронных колебаний следующие выражения: 
в секторе 1

$$
\begin{aligned}
& \Phi_{z 1}(s)=\frac{r}{\sin \mu_{z}}\left\{\left(\tau_{12}+\tau_{21}-\frac{\tau_{12} \tau_{21} v_{2}}{\varkappa}\right) \frac{s^{2}}{r^{2}}+\right. \\
& +\left[\left(\tau_{21}-\tau_{12}\right) \frac{v_{2}}{x}-\left(\tau_{12}+\tau_{21}\right) v_{1}+\frac{\tau_{12} \tau_{21} v_{1} v_{2}}{x}\right] \frac{s}{r}+ \\
& \left.+v_{1}+\frac{v_{2}}{x}-\frac{\tau_{21} v_{1} v_{2}}{x}\right\} \quad\left(0 \leqslant s \leqslant r v_{1}\right) \text {, }
\end{aligned}
$$

в секторе 2

$$
\begin{aligned}
& \Phi_{z 2}(s)=\frac{r}{\sin \mu_{z}}\left\{\left(\tau_{12}+\tau_{21}-\tau_{12} \tau_{21} v_{1}\right) \frac{s^{2}}{r^{2}}+\right. \\
& +\left[\left(\tau_{12}-\tau_{21}\right) v_{1}-\left(\tau_{12}+\tau_{21}\right) \frac{v_{2}}{x}+\frac{\tau_{12} \tau_{21} v_{1} v_{2}}{x}\right] \frac{s}{r}+ \\
& \left.+v_{1}+\frac{v_{2}}{x}-\frac{\tau_{12} v_{1} v_{2}}{x}\right\} \quad\left(0 \leqslant s \leqslant \frac{r v_{2}}{x}\right) \text {, }
\end{aligned}
$$

причем согласно (6) и (22) для $\cos \mu_{z}$ остается в силе ранее известная формула

$$
\cos \mu_{z}=1-\frac{\tau_{12}+\tau_{21}}{2}\left(v_{1}+\frac{v_{2}}{x}\right)+\frac{\tau_{12} \tau_{21} v_{1} v_{2}}{2 \varkappa}
$$

\section{3. Обсуждение результатов}

Сравнивая формулы (23) и (27) с соответствующими формулами работы ['], легко убедиться, что здесь нам удалось представить к.м.ф.Ф. в более простой форме, чем в указанной статье, а именно в форме, показывающей явную зависимость от основных параметров $r, v_{1}, v_{2}, \tau_{12}$ и $\tau_{21}$, характеризующих равновесную орбиту.

Из (23) и (27) непосредственно вытекает, что к.м.ф.Ф., а следовательно и огибающая траектории частиц в секторном циклотроне не является, в отличие от ускорителей без краевой фокусировки, симметричной относительно середины секторов. В частности, на концах секторов получим следующие различные значения к.м.ф.Ф.:

$$
\left.\begin{array}{l}
\Phi_{r 1}(0)=\Phi_{r 2}\left(\frac{r v_{2}}{\varkappa}\right)=\frac{r}{\sin \mu_{r}}\left(s_{1} c_{2}+\frac{c_{1} s_{2}}{x}+\frac{s_{1} s_{2}}{\varkappa} \tau_{21}\right) \\
\Phi_{r 1}\left(r v_{1}\right)=\Phi_{r 2}(0)=\frac{r}{\sin \mu_{r}}\left(s_{1} c_{2}+\frac{c_{1} s_{2}}{\varkappa}+\frac{s_{1} s_{2}}{\varkappa} \tau_{12}\right) \\
\Phi_{z 1}(0)=\Phi_{z 2}\left(\frac{r v_{2}}{x}\right)=\frac{r}{\sin \mu_{z}}\left(v_{1}+\frac{v_{2}}{x}-\frac{v_{1} v_{2}}{x} \tau_{21}\right) \\
\Phi_{z 1}\left(r v_{1}\right)=\Phi_{z 2}(0)=\frac{r}{\sin \mu_{z}}\left(v_{1}+\frac{v_{2}}{x}-\frac{v_{1} v_{2}}{x} \tau_{12}\right) .
\end{array}\right\}
$$

В качестве иллюстрации к (29) и (30) рассмотрим случай, когда $\tau_{12}>0$ и $\tau_{21}<0$. Согласно (7) и $(25)$, условие $\tau_{12}>0$ соответствует радиальной дефокусировке и вертикальной фокусировке краевым магнитным полем, а условие $\tau_{21}<0$ - радиальной фокусировке и вертикальной 
дефокусировке краевым магнитным полем. Поскольку, согласно (6), заключенные в скобки (после второго знака равенства) части выражений (29) и (30) являются в случае устойчивого движения положительными, то получим следующую закономерность: к.м.ф.Ф. у граничной линии, где имеется фокусировка краевым магнитным полем, превышает к.м.ф.Ф. у граничной линии, где имеется дефокусировка краевым магнитным полем.

В заключение отметим, что расчеты в разделе 1 сделаны с такой степенью общности, что позволяют найти к.м.ф.Ф. и в том случае, когда в секторах имеется слабо неоднородное магнитное поле. В этом случае можно допустить, что уравнения бетатронных колебаний в переходных областях малой длины сохраняют прежнюю форму, а в секторах надо положить:

$$
\begin{aligned}
& g_{r}(s)=\left\{\begin{array}{ll}
\frac{1-n_{1}}{r^{2}} & \text { в секторе 1 } \\
\frac{\left(1-n_{2}\right) x^{2}}{r^{2}} & \text { в секторе 2 }
\end{array}\right\} \\
& g_{z}(s)=\left\{\begin{array}{ll}
\frac{n_{1}}{r^{2}} & \text { в секторе 1 } \\
\frac{n_{2} x^{2}}{r^{2}} & \text { в секторе 2, }
\end{array}\right\}
\end{aligned}
$$

где $n_{1}$ и $n_{2}-$ показатели магнитного поля ${ }^{[8]}$ в секторах 1 и 2 .

Сравнивая (31) и (32), с одной стороны, и (7) и (25) - с другой, мы убедимся, что к.м.ф.Ф. для секторного циклотрона со слабо неоднородным полем можно в случае необходимости легко получить, если в формулах раздела 1 сделать подстановку (табл. 3):

\begin{tabular}{|c|c|c|c|c|c|c|c|c|c|c|}
\hline \multirow{2}{*}{\multicolumn{3}{|c|}{ 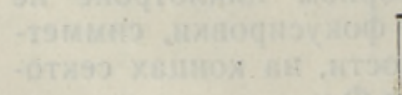 }} & \\
\hline & & & $m_{1}$ & $k_{1}$ & $m_{2}$ & $k_{2}$ & $t_{12}$ & $t_{21}$ & $\Theta_{1}$ & $\Theta_{2}$ \\
\hline \multirow{2}{*}{$\begin{array}{c}\text { Радиаль- } \\
\text { ные коле- } \\
\text { бания }\end{array}$} & Сектор & 1 & $\sqrt{1-n_{1}}$ & 1 & $\sqrt{1-n_{2}}$ & $x$ & $\tau_{12}$ & $\tau_{21}$ & $v_{1}$ & $v_{2}$ \\
\hline & Сектор & 2 & $\sqrt{1-n_{2}}$ & $x$ & $\sqrt{1-n_{1}}$ & 1 & $\tau_{21}$ & $\tau_{12}$ & $v_{2}$ & $v_{1}$ \\
\hline Верти- & Сектор & 1 & $\sqrt{n_{1}}$ & 1 & $\sqrt{n_{2}}$ & $x$ & $-\tau_{12}$ & $-\tau_{21}$ & $v_{1}$ & $v_{2}$ \\
\hline $\begin{array}{c}\text { кальные } \\
\text { колебания }\end{array}$ & Сектор & 2 & $\sqrt{n_{2}}$ & $x$ & $\sqrt{n_{1}}$ & 1 & $-\tau_{21}$ & $-\tau_{12}$ & $v_{2}$ & $v_{1}$ \\
\hline
\end{tabular}

Таблица 3

Рассмотренные примеры свидетельствуют о том, что метод [7] является весьма удобным методом вычисления к.м.ф.Ф. 


\section{Л И ТЕ РА Т Р РА}

1. Л ем б р а Ю., Изв. АН Эст. ССР. Сер. физ.-матем. и техн. наук, 11, № 1, 50 (1962).

2. Балдин А. М., Мих айлов В. В., Р аб инович М. С., ЖЭТФ, 31, 993 (1956).

3. Р а б и н о в ич М. С., Тр. ФИАН, 10, 23 (1958).

4. Мороз Е. M., Р абинович M. C., Proc. CERN Symp., 1, 547 (1956).

5. Мороз Е. М., Р абинович М. С., Приборы и техника эксперимента, № 1, 15 (1957).

6. Мороз Е. М., Р а б и но в и ч М. С., Приложение № 4 к журналу «Атомная энергия», 27 (1957).

7. Л ем 6 р а Ю. Я., ЖТФ, № 3 (1965).

8. Коломен ский А. А., Л ебеде в А. Н., Теория циклических ускорителей, Физматгиз, 1962.

Тартуский государственный университет

Поступила в редакцию

14/VII 1964

\section{J. LEMBRA}

\section{SEKTORILISE TSOKLOTRONI BETATRONVONKUMISTE VORRANDI FLOQUET' FUNKTSIOONI MOODULI RUUDU ARVUTAMISEST}

\section{Resümee}

Kiirendaja magnetilise süsteemi perioodilisuse elemendi maatriksi meetodil $\left.{ }^{7}\right]$ on tuletatud lihtsad valemid (23) ja (27) Floquet' funktsiooni mooduli ruudu arvutamiseks betatronvõnkumiste vőrrandile sektorilises tsüklotronis, mille magnetilise süsteemi perioodilisuse element koosneb kahest sektorist homogeense magnetvälja erinevate väärtustega. Saadud valemist selgub Floquet' funktsiooni mooduli ruudu vahetu sõltuvus sektorilise tsüklotroni tasakaalulist orbiiti iseloomustavatest parameetritest. On esitatud arvutusmeetodi üldistus nõrgalt mittehomogeense magnetvälja juhul.

Tartu Riiklik Olikool

Saabus toimetusse

14. VII 1964

\section{J. LEMBRA}

\section{ON THE CALCULATION OF THE SQUARE OF MODULUS OF FLOQUET'S FUNCTION FOR THE EQUATION OF BETATRON OSCILLATIONS IN CYCLOTRON WITH SECTIONED MAGNETIC SYSTEM}

\section{Summary}

The matrix method $\left[{ }^{7}\right]$ of the element of periodicity of the magnetic system of an accelerator is used for the derivation of simple formulae (23) and (27) for the calculation of the square of modulus of Floquet's function for the equation of betatron oscillations in the cyclotron with sectioned magnetic system, whose element of periodicity consists of 2 sections with different values of a homogeneous magnetic field. It is shown that the square of modulus of Floquet's function depends on the parameters characterizing the equilibrium orbit. A generalization for the case of a slightly inhomogeneous magnetic field is given.

\section{Tartu State University}

